\title{
Causal Factors of Effective Psychosocial Outcomes in Online Mental Health Communities
}

\author{
Koustuv Saha \\ Georgia Tech \\ koustuv.saha@gatech.edu
}

\author{
Amit Sharma \\ Microsoft Research India \\ amshar@microsoft.com
}

\begin{abstract}
Online mental health communities enable people to seek and provide support, and growing evidence shows the efficacy of community participation to cope with mental health distress. However, what factors of peer support lead to favorable psychosocial outcomes for individuals is less clear. Using a dataset of over $300 \mathrm{~K}$ posts by $\sim 39 \mathrm{~K}$ individuals on an online community TalkLife, we present a study to investigate the effect of several factors, such as adaptability, diversity, immediacy, and the nature of support. Unlike typical causal studies that focus on the effect of each treatment, we focus on the outcome and address the reverse causal question of identifying treatments that may have led to the outcome, drawing on case-control studies in epidemiology. Specifically, we define the outcome as an aggregate of affective, behavioral, and cognitive psychosocial change and identify Case (most improved) and Control (least improved) cohorts of individuals. Considering responses from peers as treatments, we evaluate the differences in the responses received by Case and Control, per matched clusters of similar individuals. We find that effective support includes complex language factors such as diversity, adaptability, and style, but simple indicators such as quantity and immediacy are not causally relevant. Our work bears methodological and design implications for online mental health platforms, and has the potential to guide suggestive interventions for peer supporters on these platforms.
\end{abstract}

\section{Introduction}

Online Mental Health Communities (OMHCs) are dedicated online support platforms aimed at aiding individuals to share, discuss and solicit information and support related to mental health. In many ways, OMHCs function like the online analog of support groups (Potts 2005). Anonymity and social connectedness in OMHCs help individuals overcome stigma and make candid self-disclosures about their mental health concerns (Andalibi et al. 2016). Examples of OMHCs include mental health subreddits on Reddit, condition-specific discussion forums on 7Cups, and social network-based interactions on Talklife (Pruksachatkun et al. 2019). OMHCs help individuals draw psychosocial benefits that help them cope with their mental health struggles (Love et al. 2012).

Given the growing popularity of OMHCs, research has studied various aspects of participation in these communi-

Copyright (C) 2020, Association for the Advancement of Artificial Intelligence (www.aaai.org). All rights reserved. ties and how they may lead to better psychosocial outcomes for individuals. Extending evidence from experiments that demonstrate the efficacy of online support (Winzelberg et al. 2003), studies on Reddit and TalkLife find that they offer a thriving, global community for people to talk about their mental health (Pendse et al. 2019). They provide a fine-grained data source to understand how people express mental health distress and support each other in the real world, such as shifts in suicidal ideation (De Choudhury et al. 2016).

However, relatively little attention has been directed on the peer supporters on such platforms and how they can be more effective at providing support. A natural question to ask is what kinds of supportive behavior leads to better outcomes for individuals receiving support. Identifying support characteristics in responses and discussions that lead to positive psychosocial outcomes can yield insights on the best strategies of providing support, complementing work in psychotherapy literature (Norcross and Lambert 2018). Further, by focusing on natural conversations in situ, these insights can help OMHC owners design recommendations for their members to make more effective supportive responses.

To investigate the factors that contribute to effective support, we adopt the "case-control" study design from epidemiology (Schulz and Grimes 2002). The idea is to identify individuals who have had positive outcomes (Case group) and then retrospectively compare their characteristics with a similar Control group of individuals. Specifically, we identify people who have had long-term positive psychosocial changes and compare the characteristics of responses they received to that received by those who did not have such positive changes. In the language of causal inference, each response from a peer supporter can be considered as an intervention for an individual, and we are interested to find the characteristics of interventions that lead to the maximum positive change. Using the symmetry of the back-door method (Pearl 2009), we argue that looking for interventions that vary significantly between case and control groups translate to finding interventions with causal effects on the outcome.

Specifically, we work with a longitudinal dataset of $\sim 39 \mathrm{~K}$ individuals on TalkLife, an online mental health platform. We quantify their psychosocial outcomes as an aggregate measure of affective, behavioral, and cognitive outcomes. On the basis of psychosocial change from when they joined to the present, we obtain two separate cohorts of individuals 
- psychosocially most (Case) and least (Control) improved. We compare the differences in responses across a range of characteristics drawn on psychotherapy literature like adaptability, immediacy, diversity, emotionality, language style, and nature of support. Confirming past work, we find complex linguistic attributes such as adaptability, diversity, and style are significant factors for driving positive psychosocial change. In particular, factors related to adaptability such as topical congruence and linguistic accommodation have the highest difference between Case and Control groups. Somewhat surprisingly, the average length of responses has a substantial positive effect towards driving people to better outcomes, possibly as a proxy for the linguistic factors described above. Other simple factors, however, such as number of responses and immediacy of receiving a response do not have significant differences between Case and Control.

Compared to previous work by De Choudhury and Kiciman (2017) estimating effects of using specific phrases in responses, our work has an advantage whenever one is interested in analyzing continuous treatments and finding the interventions that lead to desired outcomes. This is because most forward causal inference methods (Gelman and Imbens 2013) require binarization of treatment variables. In contrast, case-control methods avoid apriori binarization of complex treatments and estimate the differences in treatment instead. Especially in OMHCs where everyone typically receive responses, our proposed method is useful to determine the necessary dosage increase of support treatments that can increase the likelihood of positive outcomes (Hernan and Robins 2010). There is also a computational advantage. In forward causal inference methods (Rubin 2005), one may estimate a separate propensity score model for each treatment whereas our case-control method allows estimating the differences in multiple treatments at once. We discuss the methodological and practical implications of our work towards improving support in OMHCs through recommendation-based interventions for peer supporters.

Privacy, Ethics, and Disclosure. This paper uses sourced data (licensed and consented) from TalkLife. Our work is in collaboration with TalkLife, and given the sensitivity of our work, we are committed to securing the privacy of the individuals. The dataset was accessed through secured databases with necessary privacy and ethical protocols in place, and the dataset was de-identified and no personally identifiable information was used. This paper only reports aggregated measures to prevent traceability and identifiability of individuals on the platform. Even accounting for the benefits, we recognize the potential misuses, risks, and ethical consequences involved with this kind of research, which we elaborate in Discussion. This work is approved by the Institutional Review Board at Microsoft Research.

\section{Background and Related Work}

Effective Psychotherapeutic Interventions. What constitutes effective counseling and psychotherapeutic strategies has interested researchers and practitioners for a long-term now (Labov and Fanshel 1977), and treatments and therapeutic methods constantly evolve and advance over time.
Lambert and Barley (2001) formulated four areas that influence a care-seekers' outcome in psychotherapeutic settings - extratherapeutic factors, expectancy effects, specific therapy techniques, and common factors. Among these, common factors include empathy, warmth, congruence, therapeutic alliance, are found to be most highly correlated with the outcomes. In another work, Norcross and Lambert (2018) conducted a meta-analysis on the effectiveness of several elements of psychotherapeutic relationships.

In the area of online technology aided and mediated mental health interventions, Cavanagh et al. (2018) showed the efficacy of computer-mediated psychotherapy towards positive clinical outcome. Although its efficacy is yet to be established and results are mixed (Rollman et al. 2018), researchers have stressed the importance of social media as a mental health intervention platform (Ernala et al. 2017, Merolli et al. 2013, Yoo and De Choudhury 2019). Relatedly, Dinakar et al. (2015) used computational linguistics and machine learning to improve crisis counseling and interventions, Haberstroh et al. (2007) studied online counseling experiences, and Althoff et al. (2016) studied effectiveness of counseling language including adaptability, creativity, and perspective change. Our work draws upon these prior works to evaluate what factors help in desirable psychosocial outcomes in "online psychotherapeutic setting", where individuals seek and share mental health related support.

Support in Online Mental Health Communities (OMHCs). With the widespread use of social mediabased technologies, OMHCs are becoming increasingly popular (Kummervold et al. 2002, Saha et al. 2020). Originally ideated as online analogs of support groups, individuals in these communities share and seek support related to mental health concerns faced by themselves or their loved ones (De Choudhury and De 2014; Huh 2015; Saha et al. 2019a). Prior work studied how anonymity, engagement, social capital, and social connectedness help in candid self-disclosure and seeking mental health support (Andalibi et al. 2016; Ernala et al. 2018). In addition, online social support is known to build interpersonal relationships, and to improve psychological wellbeing, self-esteem, satisfaction, and reciprocity (Oh et al. 2013, Steinfield et al. 2008).

For specialized OMHC platforms such as Talklife or 7Cups, recent studies have examined positive outcomes over a sample of users (Baumel et al. 2018) or over a single thread or bursts of conversation (Kushner and Sharma 2020, Pruksachatkun et al. 2019; Pendse et al. 2019). We extend this research by focusing on long-term changes in one's mental health over a large sample of individuals and retrospectively finding the most relevant causes.

Causal Inference Studies on Observational Data The gold-standard approach to establish causality is via a randomized controlled trial. In early work, such trials were conducted to assess the efficacy of online support communities of breast cancer (Winzelberg et al. 2003). However, trials are not always feasible due to practical and ethical concerns (Hannan 2008). As an alternative, researchers resort to observational studies. While these cannot guarantee causality, observational studies allow to investigate long-term and longitudinal data, 
and are especially useful to find candidate treatments for a future randomized trial when no preferred treatment is known apriori (Rubin 2005). There are two popular means of conducting observational studies - 1) cohort based, where the treatment is known and the goal is to find its causal effects on the outcome, and 2) case-control based, where the outcomes are known, and the goal is to (retrospectively) find the treatments that potentially caused the outcome.

In our related space of social media and mental health, observational studies have examined the effects of suicidal ideation (De Choudhury et al. 2016), social support (De Choudhury and Kiciman 2017), psychiatric medications (Saha et al. 2019b), exercise (Dos Reis and Culotta 2015), alcohol use (K1c1man et al. 2018), crisis (Saha and De Choudhury 2017), and counseling interventions (Saha et al. 2018). These studies adopted the cohort based, or prospective analysis of conditioning on treatments, and matching on similar individuals to examine the differences in the outcomes, which finally quantifies the causal effects (Olteanu et al. 2017). However, cohort-based studies may not be suitable when the goal is to rank multiple continuous treatments on their causal effect, especially when almost everyone receives variable treatment dosage, and there are no obvious way to binarize the different treatments. We therefore adopt the casecontrol study design, and provide a method to analyze the effects of several continuous treatments.

\section{Data}

The dataset of our study comes from TalkLife (Pendse et al. 2019), an online mental health discussion forum, selfdescribing itself as a "safe social network to get help and give help". From the standpoint of social computing interface, TalkLife functions like many other online communities and discussion forums. The community members participate via discussion threads, where each discussion thread consists of an original post (or "post" hereon), and a number of responses by community members which are typically relevant to the post in the discussion thread. We obtain data from the TalkLife platform (in collaboration with TalkLife) from August 2011 to January 2019. This data includes discussion threads, each with a single post and a number of responses by community members. We note that TalkLife responses can also follow a hierarchical nature, however our work accounts for all kinds of responses similarly, under an umbrella term of "responses". Our dataset consists of over 6.5M posts (equal number of discussion threads) and $20 \mathrm{M}$ responses posted by over $300 \mathrm{~K}$ users. On average, each discussion thread receives 4.44 responses (stdev. $=30.60)$.

Social computing platforms tend to change significantly over the years, which could also lead to changes in usage behavior and objectives of individuals. For the purposes of our study, to minimize the effects of long-term platform-level changes and aggregated use behavior, we limit our analysis to 38,977 individuals who started participating in TalkLife after January 01, 2016, continued participation (posted more than once) beyond January 01, 2018, and overall had posted at least 15 times on the platform. Given that our data lasts till January 2019, each user has been on the platform for at most three years $($ mean=222 days, median=142 days). Given that

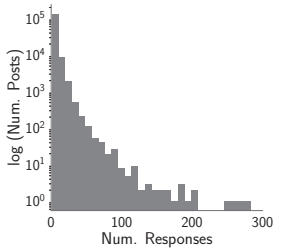

(a) Resp. per post

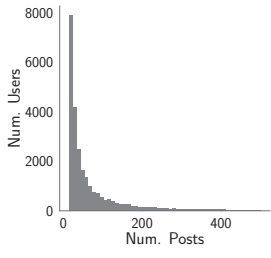

(b) Posts per user

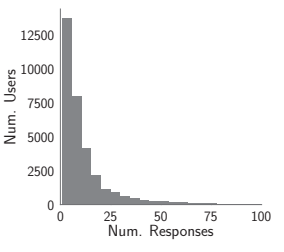

(c) Resp. per user
Figure 1: Distribution of posts and responses in study dataset.

the TalkLife platform has stabilized over recent years, we expect a lower impact of platform-level changes during this three-year time period. This study concerns a dataset comprising of 3,184,612 posts and 23,528,159 responses with 4.72 response per post $($ stdev. $=54.81)$. Figure 1 shows the distribution in our dataset as responses per post (Figure 1(a)), posts per user (Figure 1(b)), and responses per user (Figure 1(c)).

\section{Study Design and Methods Retrospective Case-Control Design}

We are interested in the effect of different kinds of supportive interventions (or treatments) on psychosocial outcomes for users on OMHCs. If interventions are well-defined (e.g., in medicine, whether a drug was prescribed), then the most common approach is to estimate the effect of each intervention separately. If $T$ is the treatment, $Y$ the outcome, and $W$ represents common causes or confounders of $T$ and $Y$, then the causal effect (Pearl 2009) of $T$ on $Y$ is represented as:

$$
\begin{aligned}
& E[Y \mid \operatorname{do}(T=1)]-E[Y \mid \operatorname{do}(T=0)] \\
& \quad=\sum_{w} E[Y \mid T=1, W]-\sum_{w} E[Y \mid T=0, W]
\end{aligned}
$$

Effectively, the method compares outcomes for people with or without the intervention while conditioning on all confounders. However, when there are multiple candidate treatments for the same outcome of interest, it may be more appropriate to ask the reverse causal question. That is, rather than the effect of a treatment, we ask about the potential causes of an observed outcome. In our problem, for example, the outcome is pre-specified-psychosocial health of individuals - but treatments are not. At one level, we could consider participation on Talklife as a means to be treated. At another level, we would like to consider the effect of several characteristics of language and behavior used by peer supporters and isolate their effects. Thus, our goal is to find treatments that lead to a significant change in the outcome.

When the outcome is well-specified, and it distinguishes two groups of Case and Control users, we can use the following estimator for finding the interventions that causally affect the outcome, based on the case-control study design in epidemiology (Schulz and Grimes 2002):

$$
\sum_{w} E[T \mid Y=1, W]-\sum_{w} E[T \mid Y=0, W]
$$

In our work, $W$ corresponds to covariates consisting of individual attributes. Intuitively, this equation refers to conditioning on $W$, and then calculating the differences between 
treatment for Case and Control. The estimator compares individuals with a positive outcome $(Y=1)$ with others $(\mathrm{Y}=0)$ and measures the difference in intervention values between the two groups, while conditioning on all known confounders. This is the so-called "reverse" causal inference problem (Gelman and Imbens 2013). Whenever there is a significant change in $T$ from $Y=0$ to $Y=1$ keeping all confounders $W$ constant, it implies that there is a causal effect of $T$ on $Y$. Given the same outcome, we analyze repeatedly to find the treatments with the highest effects. As we see later, the case-control analysis provides computational benefits especially when working with continuous intervention variables and selecting a suitable cutoff to binarize them for a future randomized experiment. Unlike cohort-based causal studies that condition on individual treatments, our approach allows accounting for a combination of treatments together on the same individual (emulating closer to the real-world).

The rest of this section contextualizes the above estimator in an OMHC. We operationalize the outcome on psychosocial health, determine the case and control groups, and then finally list potential interventions that we test.

\section{Measuring the Outcome: Psychosocial Health}

Towards our research objective of understanding effective psychotherapeutic interventions, we first operationalize "improvement in mental health outcome" as observed on TalkLife. Researchers have argued on what constitutes improvement and success in psychotherapeutic, psychological, and psychiatric care (Perkins 2001), where traditionally symptom reduction has been considered to be the improvement in quality of life. Generally speaking, "psychosocial health" is considered as an appropriate terminology that not only encompasses both psychological and social wellbeing, but also places the locus of health in the individual by including social wellbeing in the form of social adjustment and environmental response (Larson 1996). We situate our work on the impacts of social media based interventions on one's psychosocial health and wellbeing (Merolli et al. 2013). Given that psychosocial health is a complex construct and there is no easy means to quantify it, we adopt a conservative definition of psychosocial health based on observed behavior on the platform. As a user's posting behavior is our only available data, we draw upon prior work that operationalize therapeutic responses in online mental health communities and social media, grounded on psychology, psychiatry, and expressive writing literature (Ernala et al. 2017, Saha et al. 2018). We broadly group these observed psychosocial outcomes in three categories - affective, behavioral, and cognitive outcomes (Breckler 1984), and then aggregate them to construct a single outcome metric.

Affective Outcomes. Simplistically, affect refers to an emotional response, and affective behavior is indicative of one's psychological wellbeing. Because social media posts are written in a self-motivated and self-initiated fashion, language is a strong means to infer affective psychosocial health. To measure this, we use the following:

Affective Words. We use the psycholinguistic lexicon, Linguistic Inquiry and Word Count (LIWC) (Pennebaker et al. 2003) to obtain proportion of affective (positive and negative affect) keywords per user. This draws upon expressive writing literature which associate language with therapeutic symptoms. Increased use of positive affect and decreased use of negative affect words correspond with psychosocial improvement.

Language Indicative of Mental Health Symptomatic Outcomes. To identify the presence of mental health concerns, prior work built machine learning classifiers of social media language indicative of depression, anxiety, stress, suicidal ideation, and psychosis (Saha et al. 2019b). These are $n$-gram $(n=1,2,3)$ based binary SVM models. For training these classifiers, the positive class comes from domain dependent data on Reddit (r/depression, r/anxiety, r/stress, r/SuicideWatch, and $r / p$ sychosis subreddits for the corresponding classifiers), and the negative training examples come from a set of random non-mental health related content from Reddit. Similar to Saha et al. (2017; 2019b), we conduct linguistic equivalence test - cosine similarity of the word embedding representations of top $500 n$-grams in the Reddit and Talklife datasets shows a high similarity of 0.92 . This entails very similar transfer datasets, and similar performance, given that the classifiers have performed reasonably well when transferred on other social media datasets (Saha et al. 2019b). Using these classifiers, we obtain the aggregated proportion of posts that express mental health concerns corresponding to each TalkLife user. That is, lower the proportion of posts expressing mental health concerns, better is one's psychosocial health.

Behavioral Outcomes. Behavioral psychosocial health consists of an individual's overt actions, behavioral intentions, and verbal statements regarding behavior (Breckler 1984). Behaviors such as changes in social functioning and shift of interests could be indicative of an individual's changing psychological trajectory (Saha et al. 2018, Guntuku et al. 2019). To quantify behavioral psychosocial outcomes, we obtain three attributes on an individual's behavior on the platform. The first of these is activity, or the frequency of participation on the platform - this is quantified as the number of posts per day for every individual. The second is interactivity, or how interactive an individual is - this is quantified as the ratio of the number of responses (to others' posts) to the number of self-posts. This essentially quantifies an individual's behavior of providing support compared to seeking support. The final one is interaction diversity, or the topical diversity of discussions an individual engages in - each discussion thread is labeled with a particular topic (eg., relationships, family, self-harm, friends, hopes, etc.) by the original poster. These measures are directly associated with psychosocial health an increase in these measures corresponds to an improvement in psychosocial health (Saha et al. 2018).

Cognitive Outcomes. Beliefs, knowledge structures, perceptual responses, and thoughts constitute cognitive component of psychological wellbeing (Breckler 1984). Cognitive attributes is another indicator of an individual's psychological health (Bandura 1993). Drawing on psycholinguistics literature that demonstrates how the style and structure in language define one's cognitive behavior, we adopt the following measures to define cognitive psychosocial health.

Readability measures the ease with which a reader can understand a given text. We adopt the Coleman-Liau Index (CLI) which provides readability assessment based on char- 
acter and word structure within a sentence, calculated as, $C L I=(0.0588 L-0.296 S-15.8)$, where $L$ is the average number of letters per 100 words, and $S$ is the average number of sentences per 100 words. A greater CLI measure indicates a better writing quality, and an increase of CLI indicates psychosocial improvement (Ernala et al. 2017).

Complexity and Repeatability capture one's cognitive state in the form of planning, execution, and memory (Ernala et al. 2017). We quantify complexity as the average length of words per sentence, and repeatability as the normalized occurrence of non-unique words. While linguistic complexity has a positive association with one's psychosocial health, repeatability shares a negative association with the same.

Psycholinguistic Keywords. We use LIWC lexicon to obtain the proportion of keywords corresponding to cognition, perception, and linguistic style categories, where linguistic style keywords correspond to non-content keywords in language such as, temporal references (past, present, and future tense), lexical density and awareness (auxilliary verbs, preposition, adverbs, verbs, articles, conjunctions, inclusive, and exclusive), and interpersonal focus (1st person singular and plural, 2nd person, and 3rd person pronouns). Literature posits the importance of keywords in understanding cognitive behavior. For instance, the variations in pronoun use reflects the transformation in the way individuals think about themselves with respect to others, and the use of articles and adverbs could indicate how individuals process complex narratives (Pennebaker et al. 2003). A greater use of these keywords is associated with one's improved psychosocial state.

Overall Psychosocial Outcome After normalizing each of the above outcomes on a min-max scale of 0 to 1 , we operationalize psychosocial health of an individual as a composite measure of unit-weighted and sign-adjusted average across each of the outcomes so that higher values indicate a better psychosocial health (see below). As noted before, while this cannot be argued to be perfect, we believe that by accounting for several symptomatic observable changes on the platform, such a composite measure should be theoretically correlated with the actual psychosocial health of an individual.

outcome $=\operatorname{mean}\left(p a-n a-m h \_l a n g u a g e+\right.$ activity + int._diversity

+ interactivity + readability-complexity-repeatab.+cog._words)

\section{Determining Case and Control Individuals}

To understand the effects of support, social interactions, and responses (treatment), we identify and distinguish those individuals who improved the most, and those who did not after a period of time on the platform. Adopting terminologies from epidemiological observational studies, we name these groups as Case (improved) and Control (not improved or worsened) (Schulz and Grimes 2002). Ideally the improvement should be determined based on the change in mental health state in the present from their initial state on the platform (or before they are treated). As a proxy of the initial state, we quantify an individual's baseline psychosocial health on their first $n_{1}$ posts since they joined the platform. Treatment correspond to the attributes of responses received on the next $n_{2}$ posts on the platform and the outcome as the average psychosocial health over all posts after $n_{2}$. Essentially, we draw on variable treatment effect framework (Hernan and Robins

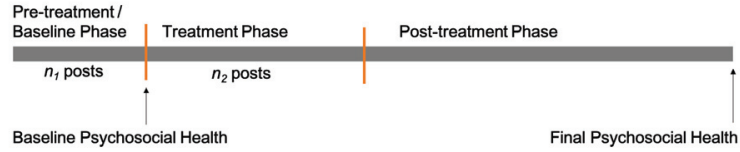

Figure 2: Schematic of a user timeline in our study design.

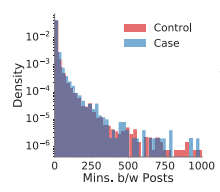

(a)

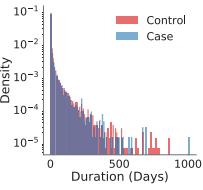

(b)

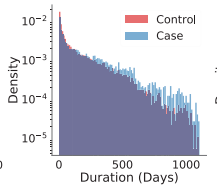

(c)

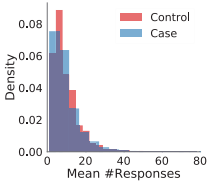

(d)
Figure 3: Distribution in Case and Control in terms of (a) time between successive posts (b) time period in treatment phase, (c) time period in post-treatment phase, (d) number of responses (per user) received in the treatment phase.

2010), and segregate an individual's timeline of activities on the platform into pre-treatment phase, treatment phase, and post-treatment phase (see Figure 2 for a schematic overview of the segregation on an individual's timeline). We choose the number of posts by a user instead of duration on the platform since it provides a better metric for exposure to responses given high variance in people's activity.

Choice of $n_{1}, n_{2}$. There is a tradeoff in choosing $n_{1}$. A smaller $n_{1}$ ensures capturing the initial state of a user without the effects of responses, but also exposes us to a high variance in estimating it. We thus vary combinations of $n_{1}$, $n_{2}$ in different values between 2 and 8 posts, and check for consistency in findings. We observe that our results are not sensitive to the choice of $n_{1}, n_{2}$. For the ease of exposition, we first discuss and report findings with pre-treatment phase of $n_{1}=3$ and treatment phase of responses received in the next $n_{2}=8$ posts per individual. We revisit the robustness of our findings for different combinations of $n_{1}, n_{2}$.

Case and Control Individuals. Within our dataset, we find that psychosocial change ranges between -0.27 and 0.36 , with a mean change of 0.04 (std.=0.05). For the purposes of our study, we define Case to be the individuals who lie in the top 80 percentile of psychosocial change, and Control to be the individuals in the bottom 50 percentile of psychosocial improvement (see Figure 4a). Our choice of $80 \%$ is motivated by the idea of restricting Case to only people with very good outcomes so that we can better understand the nature of support interventions behind those changes. With this definition, we obtain 6,789 Case and 16,972 Control individuals, whom we study for our ensuing analyses.

Testing Comparability of Case and Control Before conducting causal analysis on Case and Control, we evaluate if their data is comparable. We compare the duration between successive posts by Case and Control users (Figure 3a). We find that Case Control posts are separated by average 20 and 18 minutes respectively. There is no statistical significance as per independent sample $t$-test and Kolmogorov-Smirnov $(K S)$ test $(p>0.1)$. Given that our study design rests upon a 


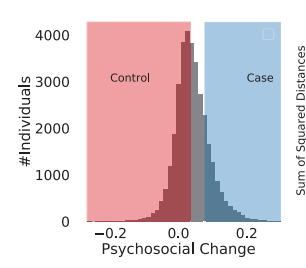

(a) Outcome Changes

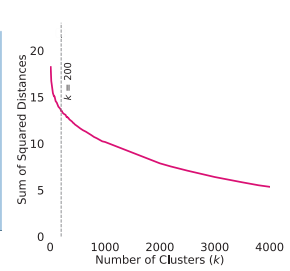

(b) Optimal $k$

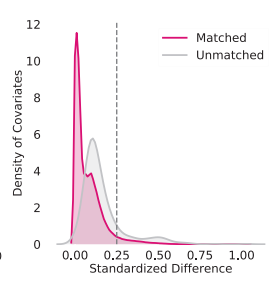

(c) Matching Balance
Figure 4: (a) Dist. of Psychosocial outcome of all individuals, (b) $k$-means clustering of individuals on covariates for several $k$, (c) Standardized differences following matching.

threshold on the number of posts for specifying the treatment phase (Figure 2), we test if this specification leads to any biases in length of participation time period by comparing the distribution of time period per Case and Control individual in treatment (Figure 3b) and post-treatment (Figure 3c) phases. The mean length of time in the treatment-phase is 15 days for Case and 16 days for Control individuals, and the same in the post-treatment phase is 221 days for Case and 204 days for Control individuals. For both comparisons we find no statistical significance as per $t$-test and $K S$-test $(p>0.1)$. Again, because we consider receiving responses as treatment, we compare if Case and Control received different number of responses overall, where we find no statistical significance as per $t$-test and $K S$-test $(p>0.1)$. These tests reveal that Case and Control datasets are comparable with minimal biases.

\section{Matching of Similar Individuals}

We next aim to identify the causes of post-treatment psychosocial outcomes. We adopt a case-control framework, that conditions on the outcomes to differentiate the treatment between Case and Control. Theoretically, given two similar individuals, their likelihood to improve is similar if they were subjected to the same treatment. Thus, outcome difference is potentially caused by the differences in treatment, provided the biases due to confounders are minimized. We assume that all potential interventions are via responses on TalkLife, or more generally that interventions outside the platform similarly affect both Case and Control.

Covariates. To reduce biases associated with confounders, the first step involves identifying a suitable set of covariates. The covariates include the exact same affective, behavioral and cognitive measures of psychosocial health, as described in the previous subsection on outcomes. However, while the outcomes are measured over posts that come after the first $n_{1}+n_{2}$ posts, the distinction is that we compute these covariates for matching using only their first $n_{1}$ posts. In effect, we control for covariates that are baseline behavioral and psychological attributes of individuals. For each covariate, we quantify an aggregated measure per individual within their pre-treatment (first $n_{1}$ ) posts and responses received to them. The covariates include their pre-treatment psychosocial measures, which are: affective measures (normalized quantity of affective words and classifiers of depression, anxiety, stress, psychosis, and suicidal ideation), behavioral measures (activity, interactivity, and interaction diversity), and cognitive
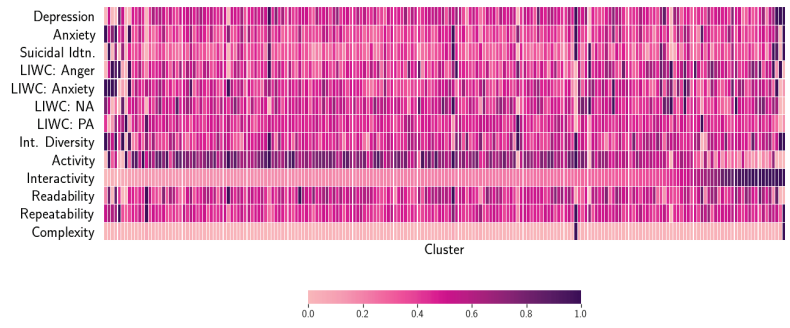

Figure 5: Heatmap showing mean values for a sample of covariates across 200 clusters. Values are rescaled using minmax scaling of 0 to 1 within a covariate.

measures (readability, complexity, and repeatability). The covariates additionally include the top $500 n$-grams $(n=1,2,3)$ per user, and the pre-treatment average number of responses received per post per individual. The choice of covariates is motivated by prior work (K1c1man et al. 2018, Saha et al. 2019b). We use these covariates as features in clustering similar users in our ensuing matching step.

Matching Approach. To find statistically comparable individuals, we use matching. This simulates a randomized trial setting by conditioning on as many as covariates as possible (Rubin 2005). To compare against counterfactual scenarios, for those who improved (Case) we find their similar (matched) counterparts among the ones who did not improve (Control). Typically, matching methods match individuals on the basis of the likelihood of being treated, however, in our case every individual is treated (or exposed to responses), although the "dosage" of treatment measures may vary. To account for variable treatment across individuals (Hernan and Robins 2010), we match individuals in an unsupervised fashion using $k$-means clustering. This approach functions like a stratified matching approach (K1c1man et al. 2018), where each cluster (or stratum) consist of matched individuals.

To determine the number of clusters $(k)$ in $k$-means, we use the well-adopted elbow heuristic — optimal $k$ can be located around the greatest drop in density across clusters. Figure $4 \mathrm{~b}$ plots the sum of squared distances of samples to the nearest cluster centroids for $k$ varying between 1 and 5,000. Manually inspecting Figure 4b and using Kneedle algorithm (Satopaa et al. 2011), we approximate that the greatest drop (maximum curvature) occurs at around $k=200$, which we adopt as the number of clusters in our analysis.

After we cluster similar individuals, we drop those clusters without sufficient number of Case and Control users as these clusters could lead to biased findings (Kiciman et al. 2018). Using a threshold of at least 10 Case and 10 Control user per cluster, we obtain 181 usable clusters that contain 6,758 Case and 16,920 Control individuals in total - together $99.6 \%$ of the Case-Control users that we identified. Each cluster essentially contains similar Case and Control individuals conditioned on pre-treatment covariates (psychosocial health and language on the platform). For better interpretation, we label the clusters by ranking on average interactivity of cluster members, i.e., those with greater interactivity are more likely to be placed at a higher value cluster. For a sample of 
interpretable covariates, Figure 5 shows cluster differences - e.g., consider the pair of first two clusters, the first cluster shows higher anxiety and interaction diversity, whereas the second has higher suicidal ideation, anger, and activity.

Evaluating Balance. The purpose of matching is to ensure that confounders are minimized to the maximum extent due to individual differences, and to help conduct like-for-like comparisons. We evaluate the balance of the covariates using standardized mean differences (SMDs) across covariates in Case and Control groups. Two groups are considered to be balanced if the covariates reveal SMD lower than 0.25 (K1ciman et al. 2018) - a condition not fulfilled in only $0.15 \%$ cases (364 out of 207,844 covariate-cluster combinations). Further, a significant drop (57\%) in mean SMD from $0.16(\mathrm{sd}=0.13)$ in the unmatched dataset to $0.07(\mathrm{sd}=0.08)$ in the matched dataset, indicates a good balance (Figure 4c).

\section{Potential Causes of Psychosocial Outcomes}

We now study the factors that potentially contribute to psychosocial changes. Below we list potential treatment measures that are based on the literature as contributors to psychosocial change. We hypothesize that these factors, both implicit and explicit in the responses contribute to an individual's psychosocial outcomes. Some of these factors are likely to be correlated among each other, aligning with the fact that "causes" of ones' psychosocial outcomes are inherently coarse and complex combination of these factors.

Number of Responses. We hypothesize that Case individuals received greater number of responses to their concerns. We rationalize that receiving more responses is associated with greater social support and sense of belonging in community, which have been found to be effective in an individual's psychosocial improvement in psychology literature (Glass and Maddox 1992). To test this hypothesis, for every individual, we calculate the average number of responses received per post in the treatment phase, and compare these averages in the matched samples of Case and Control individuals.

Verbosity. Along the lines of the above, we hypothesize that Case individuals received more verbose or longer responses to their concerns. We compare the length of responses in terms of the number of words and number of unique words received by the Case and Control individuals.

Immediacy. Because immediate and sooner responses are generally recommended in the cases of mental health crisis (Flannery and Everly 2000), we hypothesize that Case individuals received more immediate responses. Essentially, we computed the average time to the first response received by the Case and Control individuals.

Diversity/ Creativity. Drawing on the efficacy of counseling and psychotherapy styles (Althoff et al. 2016, Norcross and Lambert 2018), we hypothesize that Case individuals received more diverse responses, in comparison to the Control individuals who received more templated and generic responses. To examine this, we obtain the lexico-semantic diversity within the responses received by the Case and Control individuals. In particular, we use the 300-dimensional word embedding vector representations (Mikolov et al. 2013). For the responses in either of Case or Control corpus, we find their average cosine distance from the centroid of the corresponding corpus. This operationalizes the diversity in responses within Case and Control corpuses.

Emotionality. We hypothesize that Case individuals received responses that contained greater emotions and positive affirmations (Norcross and Lambert 2018). For this, we use LIWC to obtain the normalized occurrences of affective keywords in the responses received by the Case and the Control individuals (Pennebaker, Mehl, and Niederhoffer 2003).

Adaptability. We hypothesize that Case individuals received responses that were more customized and attuned to their concerns. This draws upon literature postulating that adaptable and linguistically accommodating responses are more effective in support than templated or generic responses (Althoff et al. 2016; De Choudhury and Kiciman 2017). Better adaptability aids improved social feedback, solidarity, social exchanges, and reciprocated feelings of intimacy (Ferrara 1991). We examine adaptability in two measures, topical congruence and linguistic style accommodation.

Topical Congruence. Motivated by Pennebaker et al.'s (2003) work that content words are indicative of numerous psychosocial aspects, we extract the content words in responses and posts (using LIWC). We operationalize topical congruence between a response and the original post as the lexicosemantic similarity between the two (Tan et al. 2016), for which we obtain the cosine similarities between their word embedding representations (Mikolov et al. 2013).

Linguistic Style Accommodation. We obtain linguistic style accommodation of each query to by using Linguistic Style Matching (Sharma and De Choudhury 2018). We compute the cosine similarity of each response and original post on the normalized occurrences of non-content or linguistic style dimensions - these are function words across the categories of articles, prepositions, pronouns, auxiliary verbs, conjunctions, adverbs, negations, etc (Pennebaker et al. 2003).

Credibility of the Responders. People tend to show trust in more credible and reputable individuals in the community (Ma et al. 2019). Accordingly, we hypothesize that Case individuals received responses from those who are experienced "care and support" givers in the community. To measure responders' experience of providing support on the platform, we quantify their tenure (or duration of time spent), interactivity (ratio of number of responses to number of posts), and activity (number and rate of posting) on TalkLife.

Language Style of Responses. Literature posits the importance of language style in effective psychotherapy (Norcross and Lambert 2018). Using personal opinion induces a sense of belonging, and also corresponds to mindful genuineness on the part of the peer-supporter. The nature of communication is a direct correlate of the complexity of language (Kolden et al. 2011). Language style can be characterized as categorical and dynamic (Pennebaker et al. 2014). Theoretically, categorical language includes approaching the world in a relatively logical, complex, and "amateur scientist" manner, and dynamic language is typically used by those who are more socially engaged, tell stories, and pay more attention to the world around them. We hypothesize that "responses received by Case individuals is more dynamic". We adopt the measure of categorical-dynamic index (CDI) proposed by Pennebaker et al. (2014). This is a bipolar index, where higher CDI indi- 
cates a categorical style, and lower CDI indicates a dynamic or narrative style. CDI for a given text is quantified on the percentage of words per style related parts of speech:

$\mathrm{CDI}=(30+$ article + preposition - personal pronoun impersonal pronoun - aux. verb - conjunction - adverb negation)

Nature of Support. Past work suggests that social support is greatly effective in helping individuals cope with mental health struggles (Kummervold et al. 2002). Situated in the "Social Support Behavioral Code", two forms of support that have received theoretical and empirical attention are emotional and informational support. Emotional support corresponds to language containing empathy, encouragement, and kindness, and is considered to be most effective form of psychosocial support (Sharma and De Choudhury 2018). Informational support, corresponds to providing information and advice, and is also known to be effective in positively impacting perceived empathy (Nambisan 2011). We hypothesize that Case individuals received greater support.

We obtain emotional (ES) and informational (IS) support in responses. We use a labeled dataset of suppotive responses on Reddit with degree of ES and IS, built and expert-verified in Sharma and De Choudhury (2018). We build two binary SVM classifiers with linear kernel, either of which characterizes the degree (high/low) of ES or IS in a post. The $k$-fold cross validation $(k=10)$ accuracy of ES and IS classifiers are 0.71 and 0.77 respectively (Table 1 ). While the classifiers are expected to perform well, better accuracy can be achieved with sophisticated models and expert annotation on TalkLife, our objective here is to leverage the feasibility of measuring supportive language. Similar to the mental health classifiers, support classifiers are also transferred from Reddit to TalkLife data, and the language similarity between the two datasets ensures a reliable transfer (Section 4). We use these classifiers to machine label all responses $-15 \%$ responses contain ES and 3.3\% responses contain IS, and then compare their prevalence per Case and Control group.

\section{The Effect of Supportive Interventions}

Per previous section, we now test the hypotheses to quantify the differences per treatment across the matched samples of Case and Control individuals. We obtain effect size (Cohen's $d$ ), and evaluate statistical significance in differences using independent sample $t$-test. We conduct KolmogorovSmirnov $(K S)$ test which essentially tests against the null hypothesis that the distributions of treatments in the Case and Control groups are drawn from the same distribution. Table 2 summarizes these differences, which we discuss here.

Number of Responses Figure 3d shows the distribution of number of responses received by Case and Control individuals in the treatment period. While on an average, Case individuals receive $25 \%$ more responses than their matched Control individuals, we find no significant differences in the number of responses received by the matched Case and Control individuals. This suggests that individuals with similar concerns, and psychological and social attributes (because of the matching framework), are likely to receive similar number of responses. Therefore, we cannot reject the null

\begin{tabular}{|c|c|c|c|c|}
\hline \multirow[b]{2}{*}{ Metric } & \multicolumn{2}{|c|}{ Emo. Support } & \multicolumn{2}{|c|}{ Inf. Support } \\
\hline & Mean & Max. & Mean & Max. \\
\hline Precision & 0.71 & 0.78 & 0.73 & 0.87 \\
\hline Recall & 0.71 & 0.80 & 0.77 & 0.87 \\
\hline$\overline{\mathrm{F}} \overline{1}$ & $\overline{0.70}$ & $0.7 \overline{7}$ & $\overline{0} . \overline{7} \overline{3}$ & $\overline{0.87}$ \\
\hline Accuracy & 0.71 & 0.78 & 0.77 & 0.87 \\
\hline AUC & 0.79 & 0.91 & 0.82 & 0.92 \\
\hline
\end{tabular}

Table 1: Accuracy metrics of the Support Classifiers

\begin{tabular}{|c|c|c|c|c|c|}
\hline Measure & Case & Control & $d$ & $t$ & KS \\
\hline Num. Responses & 16.34 & 12.98 & 0.09 & 0.88 & 0.13 \\
\hline \multicolumn{6}{|l|}{ 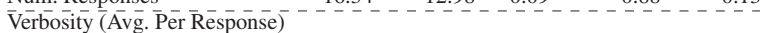 } \\
\hline Num. Words & 19.98 & 18.07 & 0.96 & $9.08^{* * *}$ & $0.43 * * *$ \\
\hline Num. Unique Words & 17.60 & 16.19 & 0.97 & $9.20 * * *$ & $0.48 * * *$ \\
\hline $\bar{I}$ Immēiacy (Minutes) & $\overline{6} . \overline{2} 2^{-}$ & $-5.9 \overline{5}$ & $0 . \overline{11}$ & $\overline{1} . \overline{05}$ & $0 . \overline{0} \overline{9}$ \\
\hline Diversity/Creativity & 0.66 & 0.63 & 1.09 & $9.18 * * *$ & $0.46 * * *$ \\
\hline \multicolumn{6}{|l|}{ Ēmotionality (\% Words) } \\
\hline Anger & 0.71 & 0.70 & 0.05 & 0.43 & $0.16^{*}$ \\
\hline Anxiety & 0.33 & 0.31 & 0.15 & 1.41 & 0.12 \\
\hline Sadness & 0.48 & 0.47 & 0.08 & 0.73 & 0.13 \\
\hline Neg. Affect & 0.87 & 0.87 & 0.00 & 0.02 & 0.11 \\
\hline Pos. Affect & 5.67 & 5.49 & 0.21 & $1.91 * *$ & $0.12 * *$ \\
\hline Swear & 0.36 & 0.36 & 0.04 & 0.38 & $0.17 *$ \\
\hline \multicolumn{6}{|l|}{$\bar{A} \bar{d}$ ap̀tābility } \\
\hline Topical Congruence & 0.65 & 0.61 & 1.22 & $11.55^{* * *}$ & $0.50 * * *$ \\
\hline Linguistic Accommodation & 0.80 & 0.61 & 1.38 & $18.14 * * *$ & $0.63 * * *$ \\
\hline \multicolumn{6}{|l|}{$\bar{C}$ rédibility of $\bar{R}$ esponders } \\
\hline Tenure (days) & 233.93 & 234.49 & -0.00 & -0.06 & 0.08 \\
\hline Interactivity & 22.46 & 19.39 & 0.32 & $2.93 * *$ & $0.13^{*}$ \\
\hline Num. Posts & 2987.34 & 2721.91 & 0.61 & $5.75^{* * *}$ & $0.40 * * *$ \\
\hline Posts per Day & 11.73 & 11.01 & 0.15 & 1.42 & 0.10 \\
\hline$\overline{\text { Language Style }}(\mathrm{CD} \overline{\mathrm{C}})$ & $\overline{3} . \overline{3} 9^{-}$ & ${ }^{-} \overline{4.10}$ & $-\overline{0.45}$ & $-\overline{3} . \overline{7} 4^{*} \bar{*} * \bar{*}$ & $-0.2 \overline{5} * \bar{*} \bar{*}$ \\
\hline \multicolumn{6}{|l|}{ Nature of Support } \\
\hline Emotional & 0.20 & 0.17 & 0.93 & $8.82 * * *$ & $0.36 * * *$ \\
\hline Informational & 0.05 & 0.04 & 0.55 & $5.20 * * *$ & $0.31 * * *$ \\
\hline
\end{tabular}

Table 2: Summary of differences in responses received by Case and Control individuals. We report average occurrences across matched clusters, effect size (Cohen's $d$ ), independent sample $t$-statistic, and $K S$-statistic. Rows with significant differences are shaded in grey, $p$-values are reported after Bonferroni correction (* $p<0.05$, ** $p<0.01$, *** $p<0.001$ ).

hypothesis that the number of responses received by Case and Control individuals are from the same distribution.

Verbosity. Case individuals receive more verbose responses than the matched Control individuals. This is revealed by both average length of response $(t=9.08, p<0.05)$, and average number of unique words per response $(t=8.91, p<0.05)$, where Case individuals receive $11 \%$ more words, and $9 \%$ more unique words per response. This supports our hypothesis on the differences in verbosity, suggesting longer responses and lower repeatability of words are more likely to help psychosocial improvement.

Immediacy. We find no significant difference in immediacy or the time to respond to posts. This could be because we study long-term and averaged-out improvements of psychosocial outcomes, rather than short-term bursts. Again, platformspecific design and post ranking on homepage plausibly does not distinguish the type and criticality of concern leading to all posts being responded back in similar intervals of time. This phenomenon is further revealed by the low standard deviation ( $\sim 12$ mins.) in the time-to-first-response across all the responses in our TalkLife dataset. That said, immediacy 
is considered to be essential for coping with critical and crisis circumstances, and this leaves room for future investigations on the prevalence of such instances on TalkLife.

Diversity/ Creativity. Supporting our next hypothesis, we find that the responses received by Case individuals are typically more diverse. The average distance (or diversity) among the responses received by the Case individuals is $5 \%$ higher $(t=9.18, p<0.05)$. This also hints at the possibility that the Control individuals received more generic and templated responses. To understand this better in context, we inspect a few top keywords in responses received by the Case and Control users, to find many generic responses such as hope great day, wish good luck, etc. in responses to Control individuals.

Emotionality. As Table 2 indicates, we find significant differences in the expression of positive affect - Case individuals received $3.5 \%$ greater positive affect. This aligns with literature that greater positivity is associated with effective psychotherapy (Truax and Carkhuff 2007). In contrast, we find no significant differences in emotionality across anger, anxiety, sadness, negative affect, and swear. Nonetheless, a common trend across all the affective attributes is that the responses received by the Case individuals show a greater occurrence than that by Control individuals. Together, our hypothesis on emotionality is only partially supported.

Adaptability We measure adaptability of the responses in terms of topical congruence and linguistic style accommodation. Topical congruence occurs $6.6 \%$ higher in the responses received by the Case individuals than the Control individuals $(t=11.55, p<0.05)$. In terms of linguistic accommodation, the responses received by the Case individuals show $31.15 \%$ greater $(t=18.14, p<0.05)$ linguistic style matching than the ones received by the Control individuals. Both the measures of adaptability, therefore, support our hypothesis, aligning with prior work that greater adaptability in responses is associated with increased supportive outcomes.

Credibility of the Responders. To examine if responder credibility significantly varied between Case and Control responses, we measure the differences in the responders' tenure (number of days on the platform), interactivity, number of posts, and the frequency of posting behavior (posts per day). Among these, we find no significant differences in the tenure and the number of posts per day. However, we find $16 \%$ greater interactivity and $10 \%$ greater number of posts for the responders to Case individuals as compared to that to the Control individuals. This suggests that responses from members who are more active on the platform seem to be typically more effective. Drawing on prior work, it may be associated with the fact that the members who are more experienced with the platform use more linguistically accommodating language or probably learn over time in what constitutes more supportive responses. Supporting our hypothesis, we find that Case individuals greatly received responses from those who are experienced "care and support" givers in the community. Language Style of Responses. We find that the average Categorical Dynamic Index (CDI) of responses received by Case individuals is $17 \%$ lower. This suggests that the responses received by Case are more dynamic in nature, or exhibit a dynamic style of thinking including a focus on others (such as greater use of pronouns), time-based stories, and use of simpler words (Pennebaker et al. 2014). Supporting our hypothesis, we conjecture that dynamic style of writing is likely to help psychosocial improvements on the platform.

Nature of Support. We find that responses to Case is higher in both emotional and informational support. Among these, Case individuals receive $18 \%$ greater emotional support, and $25 \%$ greater informational support. Therefore, our hypothesis is supported, and we conjecture that greater support contributes to better psychosocial improvement on the platform. While prior work (De Choudhury and Kicıman 2017) compared the efficacy of emotional against informational support, our work finds that both kinds of support are effective towards psychosocial improvement.

Summary. We find that many of the treatment measures positively impact long-term psychosocial outcomes. We can use Cohen's $d$ to rank treatments by their efficacy. Based on Case and Control means from Table 2, we can construct a binary treatment with a mean-split threshold on Case and Control means. This maybe interpreted as treatments with high Cohen's $d$ will likely ensure higher fraction of (binary) treated individuals with outcomes similar to the Case group. Our results indicate adaptability, diversity, verbosity, and emotional support rank highest in differentiating Case and Control individuals and thus can be considered as preferable treatment candidates for a future randomized experiment.

\section{Robustness of Findings}

Recall that our study design relies on chosen values of $n_{1}$ and $n_{2}$ posts to define pre-treatment, treatment, and posttreatment phases (Figure 2). We test if our findings hold robust for a variety of $\left(n_{1}, n_{2}\right)$ combination pairs. For different pairs, we re-conduct our entire study including measuring outcomes, conducting matching, testing balance, and computing differences in treatment for matched Case and Control. Figure 6 shows the differences as effect-size (Cohen's $d$ ) across $\left(n_{1}, n_{2}\right)$ pairs of $(3,8),(2,6),(3,5),(4,4),(5,3),(6,2)$, $(3,6)$, and $(4,6)$. We find that effect size is very similar across all combinations of $\left(n_{1}, n_{2}\right)$, showing a low standard deviation of 0.08 on average. Again, the treatment measures consistently show similar statistical significance as per $t$-test and $K S$-test. All these significant measures also show the same directionality of differences, such as verbosity, diversity, and adaptability are uniformly greater, and CDI is uniformly smaller for responses received by Case individuals.

A component of our work is the decision to separately estimate the treatment differences for each treatment, rather than considering their effects together. While we measure the differences for our theory-driven treatments independently, these treatment measures can be correlated and interdependent, e.g., positive affect and emotional support. Our study design is motivated in providing interpretative understanding of how psychotherapeutic measures function towards psychosocial improvement in OMHCs. Still, to test the robustness of independent comparisons, we build regression models with treatment measures as independent variables and overall psychosocial outcome as (continuous) dependent variable. We control our models with the same covariates used in matching, and eliminate correlated features using variance inflation factor (threshold=10) (Das Swain et al. 2019). We 


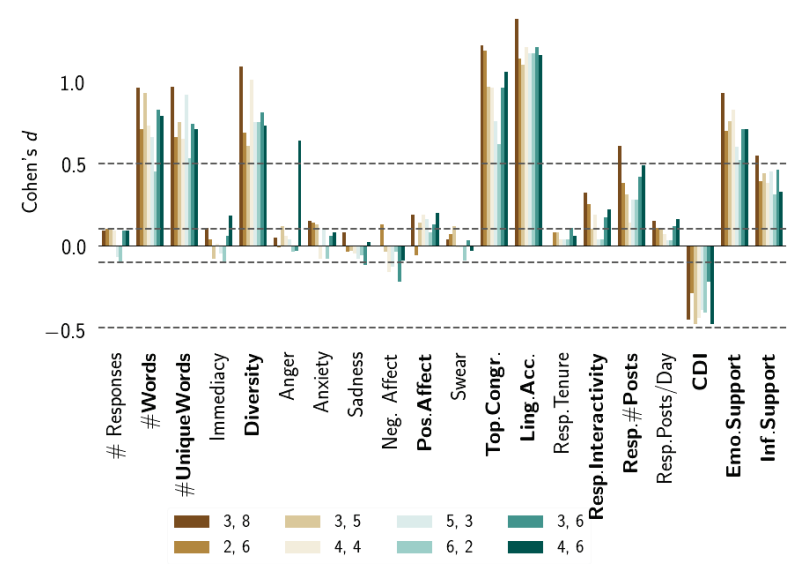

Figure 6: Cohen's $d$ of treatment differences in responses received by Case and Control users for several combinations of $\left(n_{1}, n_{2}\right)$ breakpoints defining pre- and post- treatment phases. Boldfaced measures show statistical significance in $t$-test and $K S$-test $(p<0.05)$. Statistically significant measures show consistent directionality in differences.

\begin{tabular}{|c|c|c|c|}
\hline Measure & Coefficient & Measure & Coefficient \\
\hline Num. Words & $7.32 e-5^{\star * *}$ & Topical Congruence & $2.11 \mathrm{e}-2^{\star * *}$ \\
\hline Diversity & $1.03 e-6^{* *}$ & Resp. Interactivity & $2.01 e-5^{*}$ \\
\hline EmōtionaT Suppor & $5.5 \overline{5} \mathrm{e}^{-}-\overline{3}^{\star \bar{*} *^{-}}$ & ${ }^{-} \mathrm{C} \overline{\mathrm{I}} \overline{\mathrm{I}}$ & $-\overline{3} . \overline{5} 2 \mathrm{e}^{-}-5^{\star \star}$ \\
\hline Informational Support & $8.33 e-4^{*}$ & Anger & $-4.72 \mathrm{e}-3^{*}$ \\
\hline
\end{tabular}

Table 3: Coefficients of linear regression of treatment measures as independent variables and psychosocial outcomes as dependent variables. Only statistically significant coefficients are reported $(* p<0.05$, ** $p<.01$, *** $p<0.001)$.

also include regularizations $(\mathrm{L} 1, \mathrm{~L} 2)$ and interaction terms (degree 2) in the regression models. We find all interaction terms show statistically insignificant effects. The regularized and unregularized models show similar coefficients. For linear interpretability, Table 3 reports the unregularized model's coefficients of treatment measures found to be significant. The magnitude of regression coefficients is interesting and inspires further theoretical and empirical investigations. Consistent with our previous analysis, we find that the directionality of the regression coefficients agree with that we found by independently testing the treatment measures (Table 2).

The consistency of results via different approaches confirm that our findings are robust and not sensitive to choice of treatment periods or specific estimation methods, but rather a reflection of the phenomenon in our context of TalkLife.

\section{Discussion and Conclusion}

We studied factors that contribute to psychosocial changes in OMHCs using a case-control design. We examined whether effective factors identified in psychotherapy literature are also similarly effective in OMHCs. Confirming past work, we find that factors such as diversity, adaptability, positivity, supportive nature, and dynamicity in responses are associated with effective psychosocial support. In contrast, simple factors such as immediacy and quantity of responses show insignifi- cant effects on psychosocial outcomes. Our findings can be used to rank potential interventions for peer supporters.

Methodological implications. Our work provides a useful alternative to cohort-based analyses for studying cause-andeffect in online communities, especially when the outcome is well-specified and treatments are continuous variables. In such cases, our approach can help study two dimensional changes in the treatments - 1) along the breadth, that is studying several treatments together, and 2) along the depth, that is how much of a treatment is necessary.

From a treatment dosage perspective, each measure considered in our study is a continuous variable, and it is often not possible to determine an appropriate binary cutoff for (no) treatment in a prospective causal-inference setup. Our approach avoids this limitation by focusing on the differences in the treatment measures in Case and Control groups - and suggesting the dosage of measure required for desirable outcomes across a subpopulation (Table 2). This can be useful for design interventions on a social computing platform. For instance, TalkLife can propose guidelines that recommend expectations to the members in what ways they would be helped. Also, such differences can be used to formulate treatment cutoff in conducting careful experimental studies to verify and adopt design changes. More generally, our approach allows examining several treatments that potentially contribute to the same desirable outcome. Because the effects of each treatment can vary across individuals, such a study design helps to identify which treatment or combinations of treatments could be effective for certain individuals.

Implications for OMHCs. Given that OMHCs largely rely on amateur peer supporters, one of the biggest questions is how to help supporters write more effective responses. By comparing factors that lead to a positive outcome, our work provides evidence on effective support factors. We provide a way to rank and compare potential interventions so that effective candidate treatments can be considered and encouraged. For example, based on our results, OMHCs may nudge members to write more positive or adaptive responses. Our work also contributes to digital therapeutics, given limited availability of trained psychotherapy providers, we believe insights drawn from our work can be useful to train peer-supporters and volunteers who want to help in OMHCs (Kazdin 2011; Torous and Hsin 2018). That said, causal evidence from observational studies comes with the assumption that all confounders were conditioned. As randomized experiments are the gold standard to measure efficacy, our work provides a means to prioritize which treatments to consider for such experiments of understanding effective interventions.

Towards personalized support. From the perspective of individualized and precision medicine, our work builds the case for patient-centered and personalized psychotherapeutic care (Shippee et al. 2012). We find that, just like in faceto-face settings, templated and generic responses are not as effective as personalized and adaptive responses. Applying our approach of stratifying (or clustering) individuals based on psycholinguistic and psychosocial similarity may enable decision-making on what combination of treatments can be more effective in particular clusters. This can help design frameworks to tailor treatments per cluster of individuals. 
Ethical Implications. Despite the potential, there are important ethical implications associated with using such quantitative analyses in practice. Privacy considerations should be made when machine guided interventions are tailored to OMHC participation. We expect analyses to be over deidentified datasets and interventions to be restricted to the online platform, ensuring that such analyses cannot be used to monitor one's trajectory of mental health and make offline decisions based on it. There are potential civil and ethical liability concerns in providing machine-guided support in an online medium, leaving room for further discussions on adopting these approaches in practice (Chancellor et al. 2019).

Limitations and Future Work. Our work has limitations, which also suggest promising future directions. We do not account for spill-over and passive engagement effects, e.g., individuals may be helped by browsing discussion threads of mental health support. We only consider mean-aggregated psychosocial outcomes, which is likely unable to capture shorter changes of psychosocial outcomes, e.g., individuals who show show fluctuating affect, which maybe accounted for by using complementary data sources (Morshed et al. 2019). Our operationalization does not unpack intricacies in each psychosocial outcome separately, and does not encompass all mental health conditions; certain psychosocial changes (e.g., activity) considered to be positive in our study may not be applicable in certain mental health conditions (e.g., ADHD). Future work can examine psychosocial outcomes per condition, in a fine-grained temporal fashion.

Because we examine observable behavior on TalkLife and plausibly excludes offline and latent individual differences, we cannot confirm clinical validity. Future work can obtain consented individual difference data (Saha et al. 2019) and expert-appraisal (Ernala et al. 2018), to validate these findings with greater rigor. We only study those who show continued participation on the platform. This "dropouts" issue is also encountered in experimental and trial settings (Lindsey 2000), and future work can include measures like failed interactions probability (Zhang et al. 2018) and survival analysis to account member behavior (Yang et al. 2017).

As any other observational study, we recognize that we do not infer "true causality". We cannot eliminate the likelihood of type II errors, a vulnerability of retrospective causal design. Gelman and Rubins (2013) argue that reverse-causal problems are better studied with forward-causal questions, and Watts () notes the impossibility to test all explanations simultaneously. While acknowledging these concerns, we believe our work is a step towards understanding the effects of a variety of heterogeneous factors in psychosocial outcomes. Accounting for all possible confounds is technically infeasible, and our work only minimizes the confounds by using a variety of theory-driven covariates, thereby providing insights beyond simpler correlational analyses. Alternative study designs such as instrumental variable methods may help to further minimize confounding biases. While our study only considers a finite set of treatment measures, more measures can be easily plugged in to understand their effectiveness. Our study design facilitates a simple but robust mechanism to understand the factors associated with psychosocial outcomes in an online setting, and in turn helps us draw action- able insights and implications towards running confirmatory randomized experiments and designing effective OMHCs.

\section{Acknowledgements}

Saha conducted this work while at Microsoft. We thank TalkLife for support. We thank Monojit Choudhury, Munmun De Choudhury, Daejin Choi, Sindhu Ernala, Emre Kicıman, Vedant Das Swain, Taisa Kuhner, Sachin Pendse, Ian Stewart, Adith Swaminathan, and Dong Whi Yoo for feedback.

\section{References}

Althoff, T.; Clark, K.; and Leskovec, J. 2016. Large-scale analysis of counseling conversations: An application of natural language processing to mental health. TACL.

Andalibi, N.; Haimson, O. L.; De Choudhury, M.; and Forte, A. 2016. Understanding social media disclosures of sexual abuse through the lenses of support seeking and anonymity. In Proc. CHI. Bandura, A. 1993. Perceived self-efficacy in cognitive development and functioning. Educational psychologist 28(2):117-148.

Baumel, A.; Tinkelman, A.; Mathur, N.; and Kane, J. M. 2018. Digital peer-support platform (7cups) as an adjunct treatment for women with postpartum depression: feasibility, acceptability, and preliminary efficacy study. JMIR mHealth and uHealth 6(2):e38.

Breckler, S. J. 1984. Empirical validation of affect, behavior, and cognition as distinct components of attitude. J. Pers. Soc. Psychol. Cavanagh, K.; Belnap, B. H.; Rothenberger, S. D.; Abebe, K. Z.; and Rollman, B. L. 2018. My care manager, my computer therapy and me: the relationship triangle in computerized cognitive behavioural therapy. Internet interventions.

Chancellor, S.; Baumer, E. P.; and De Choudhury, M. 2019. Who is the "human" in human-centered machine learning: The case of predicting mental health from social media. PACM HCI (CSCW).

Das Swain, V., et al. 2019. A multisensor person-centered approach to understand the role of daily activities in job performance with organizational personas. Proc. IMWUT.

De Choudhury, M., and De, S. 2014. Mental health discourse on reddit: Self-disclosure, social support, and anonymity. In ICWSM.

De Choudhury, M., and Kicıman, E. 2017. The language of social support in social media and its effect on suicidal ideation risk. In ICWSM.

De Choudhury, M.; Kiciman, E.; Dredze, M.; Coppersmith, G.; and Kumar, M. 2016. Discovering shifts to suicidal ideation from mental health content in social media. In CHI.

Dinakar, K.; Chen, J.; Lieberman, H.; Picard, R.; and Filbin, R. 2015. Mixed-initiative real-time topic modeling \& visualization for crisis counseling. In IUI.

Dos Reis, V. L., and Culotta, A. 2015. Using matched samples to estimate the effects of exercise on mental health from twitter.

Ernala, S. K.; Rizvi, A. F.; Birnbaum, M. L.; Kane, J. M.; and De Choudhury, M. 2017. Linguistic markers indicating therapeutic outcomes of social media disclosures of schizophrenia. CSCW.

Ernala, S. K.; Labetoulle, T.; Bane, F.; Birnbaum, M. L.; Rizvi, A. F.; Kane, J. M.; and De Choudhury, M. 2018. Characterizing audience engagement and assessing its impact on social media disclosures of mental illnesses. In ICWSM.

Ferrara, K. 1991. Accommodation in therapy. Contexts of accommodation: Developments in applied sociolinguistics.

Flannery, R. B., and Everly, G. S. 2000. Crisis intervention: A review. International Journal of Emergency Mental Health.

Gelman, A., and Imbens, G. 2013. Why ask why? forward causal inference and reverse causal questions.

Glass, T. A., and Maddox, G. L. 1992. The quality and quantity of social support: stroke recovery as psycho-social transition. SSM. 
Guntuku, S. C.; Buffone, A.; Jaidka, K.; Eichstaedt, J. C.; and Ungar, L. H. 2019. Understanding and measuring psychological stress using social media. In Proc. ICWSM.

Haberstroh, S.; Duffey, T.; Evans, M.; Gee, R.; and Trepal, H. 2007. The experience of online counseling. JMHC.

Hannan, E. L. 2008. Randomized clinical trials and observational studies: guidelines for assessing respective strengths and limitations. Hernan, M. A., and Robins, J. M. 2010. Causal inference.

Huh, J. 2015. Clinical questions in online health communities: the case of see your doctor threads. In Proc. CSCW.

Kazdin, A. E. 2011. Evidence-based treatment research: Advances, limitations, and next steps. American Psychologist 66(8):685.

Kiciman, E.; Counts, S.; and Gasser, M. 2018. Using longitudinal social media analysis to understand the effects of early college alcohol use. In ICWSM.

Kolden, G. G.; Klein, M. H.; Wang, C.-C.; and Austin, S. B. 2011. Congruence/genuineness. Psychotherapy 48(1):65.

Kummervold, P. E., et al. 2002. Social support in a wired world: use of online mental health forums in norway.

Kushner, T., and Sharma, A. 2020. Bursts of activity: Temporal patterns of help-seeking and support in online mental health forums. Labov, W., and Fanshel, D. 1977. Therapeutic discourse: Psychotherapy as conversation. Academic Press.

Lambert, M. J., and Barley, D. E. 2001. Research summary on the therapeutic relationship and psychotherapy outcome. Psychother.

Larson, J. S. 1996. The world health organization's definition of health: Social versus spiritual health. Social Indicators Research.

Lindsey, J. 2000. Dropouts in longitudinal studies: definitions and models. Journal of biopharmaceutical statistics 10(4):503-525.

Love, B.; Crook; et al. 2012. Exploring psychosocial support online: a content analysis of messages in an adolescent and young adult cancer community.

Ma, X.; Cheng, J.; Iyer, S.; and Naaman, M. 2019. When do people trust their social groups? In $\mathrm{CHI}$.

Merolli, M.; Gray, K.; and Martin-Sanchez, F. 2013. Health outcomes and related effects of using social media in chronic disease management: a literature review and analysis of affordances. $J$. Biomed. Inform.

Mikolov, T.; Sutskever, I.; Chen, K.; Corrado, G. S.; and Dean, J. 2013. Distributed representations of words and phrases and their compositionality. In NIPS.

Morshed, M. B.; Saha, K.; Li, R.; D’Mello, S. K.; De Choudhury, M.; Abowd, G. D.; and Plötz, T. 2019. Prediction of mood instability with passive sensing. PACM IMWUT.

Nambisan, P. 2011. Information seeking and social support in online health communities: impact on patients' perceived empathy. Norcross, J. C., and Lambert, M. J. 2018. Psychotherapy relationships that work iii. Psychotherapy 55(4):303.

Oh, H. J.; Lauckner, C.; Boehmer, J.; Fewins-Bliss, R.; and Li, K. 2013. Facebooking for health: An examination into the solicitation and effects of health-related social support on social networking sites. Comput. Hum. Behav.

Olteanu, A.; Varol, O.; and Kiciman, E. 2017. Distilling the outcomes of personal experiences: A propensity-scored analysis of social media. In Proc. CSCW.

Pearl, J. 2009. Causal inference in statistics: An overview.

Pendse, S. R.; Niederhoffer, K.; and Sharma, A. 2019. CrossCultural Differences in the Use of Online Mental Health Support Forums. PACM HCI (CSCW).

Pennebaker, J. W.; Chung, C. K.; Frazee, J.; Lavergne, G. M.; and Beaver, D. I. 2014. When small words foretell academic success: The case of college admissions essays. PloS one 9(12):e115844.

Pennebaker, J. W.; Mehl, M. R.; and Niederhoffer, K. G. 2003. Psychological aspects of natural language use: Our words, our selves. Annu. Rev. Psychol.
Perkins, R. 2001. What constitutes success?: The relative priority of service users' and clinicians' views of mental health services.

Potts, H. W. 2005. Online support groups: an overlooked resource for patients.He@lth Information on the Internet.

Pruksachatkun, Y.; Pendse, S. R.; and Sharma, A. 2019. Moments of change: Analyzing peer-based cognitive support in online mental health forums. In $C H I$.

Rollman, B. L., et al. 2018. Effectiveness of online collaborative care for treating mood and anxiety disorders in primary care: a randomized clinical trial. JAMA psychiatry.

Rubin, D. B. 2005. Causal inference using potential outcomes: Design, modeling, decisions. J. Am. Stat. Assoc.

Saha, K., and De Choudhury, M. 2017. Modeling stress with social media around incidents of gun violence on college campuses.

Saha, K., et al. 2019. Social media as a passive sensor in longitudinal studies of human behavior and wellbeing.

Saha, K.; Chan, L.; De Barbaro, K.; Abowd, G. D.; and De Choudhury, M. 2017. Inferring mood instability on social media by leveraging ecological momentary assessments. Proc. ACM IMWUT. Saha, K.; Kim, S. C.; Reddy, M. D.; Carter, A. J.; Sharma, E.; Haimson, O. L.; and De Choudhury, M. 2019a. The language of lgbtq+ minority stress experiences on social media. PACM HCI.

Saha, K.; Sugar, B.; Torous, J.; Abrahao, B.; Kıcıman, E.; and De Choudhury, M. 2019b. A social media study on the effects of psychiatric medication use. In ICWSM.

Saha, K.; Ernala, S. K.; Dutta, S.; Sharma, E.; and De Choudhury, M. 2020. Understanding moderation in online mental health communities. In HCII. Springer.

Saha, K.; Weber, I.; and De Choudhury, M. 2018. A social media based examination of the effects of counseling recommendations after student deaths on college campuses. In ICWSM.

Satopaa, V.; Albrecht, J.; Irwin, D.; and Raghavan, B. 2011. Finding a "kneedle" in a haystack: Detecting knee points in system behavior. Schulz, K. F., and Grimes, D. A. 2002. Case-control studies: research in reverse. The Lancet.

Sharma, E., and De Choudhury, M. 2018. Mental health support and its relationship to linguistic accommodation in online communities. In $\mathrm{CHI}$.

Shippee, N. D.; Shah, N. D.; May, C. R.; Mair, F. S.; and Montori, V. M. 2012. Cumulative complexity: a functional, patient-centered model of patient complexity can improve research and practice. $J$. Clin. Epidemiol.

Steinfield, C.; Ellison, N. B.; and Lampe, C. 2008. Social capital, self-esteem, and use of online social network sites: A longitudinal analysis. $J A D P$.

Tan, C.; Niculae, V.; Danescu-Niculescu-Mizil, C.; and Lee, L. 2016. Winning arguments: Interaction dynamics and persuasion strategies in good-faith online discussions. In Proc. $W W W$.

Torous, J., and Hsin, H. 2018. Empowering the digital therapeutic relationship: virtual clinics for digital health interventions. NPJ.

Truax, C. B., and Carkhuff, R. 2007. Toward effective counseling and psychotherapy: Training and practice. Transaction Publishers. Watts, D. J. Common sense and sociological explanations.

Winzelberg, A. J.; Classen, C.; Alpers, G. W.; Roberts, H.; Koopman, C.; Adams, R. E.; Ernst, H.; Dev, P.; and Taylor, C. B. 2003. Evaluation of an internet support group for women with primary breast cancer. Cancer.

Yang, D.; Kraut, R.; and Levine, J. M. 2017. Commitment of newcomers and old-timers to online health support communities.

Yoo, D. W., and De Choudhury, M. 2019. Designing dashboard for campus stakeholders to support college student mental health.

Zhang, J.; Chang, J.; Danescu-Niculescu-Mizil, C.; Dixon, L.; Hua, Y.; Taraborelli, D.; and Thain, N. 2018. Conversations gone awry: Detecting early signs of conversational failure. In Proc. ACL. 